\title{
Complications of syndesmotic screw removal
}

T. Schepers ${ }^{1,2}$, E.M.M. van Lieshout ${ }^{2}$, M.R. de Vries ${ }^{1}$, M. Van der Elst ${ }^{1}$

${ }^{1}$ Department of Surgery and Traumatology, Reinier de Graaf Groep Delft, The Netherlands

${ }^{2}$ Department of Surgery-Traumatology, Erasmus MC, University Medical Center Rotterdam, Rotterdam, The Netherlands

\section{Corresponding author:}

T. Schepers, MD PhD

Erasmus MC, University Medical Center Rotterdam

Department of Surgery-Traumatology

Room H-822k

P.O. Box 2040

3000 CA Rotterdam

The Netherlands

Tel: $+31-10-7031050$

Fax: $+31-10-7032396$

E-mail: t.schepers@erasmusmc.nl 


\section{Abstract}

Background: Currently, the metallic syndesmotic screw is the gold standard in the treatment of syndesmotic disruption. Whether or not this screw needs to be removed remains debatable. The aim of the current study was to determine the complications which occur following routine removal of the syndesmotic screw following operative treatment of unstable ankle fractures.

Materials and methods: This was a retrospective study with consecutive cases in a Level-2 Trauma center. All patients with routine removal of a syndesmotic screw, following the treatment of an unstable ankle fracture, between January 1, 2004 and November 30, 2010 were included. Complications recorded were: 1) minor or major wound infection following removal of the syndesmotic screw, 2) recurrent syndesmotic diastasis, and 3) unnecessary removal of a broken screw, not recognized during pre-operative planning prior to surgery. Results: A total of 76 patients were included. A wound infection occurred in $9.2 \%$ of which $2.5 \%$ were deep infections requiring reoperation. Recurrent syndesmotic diastasis was found in $6.6 \%$ of patients, and in $6.6 \%$ screws were broken at the time of implant removal. In the group with recurrent diastasis the screws were removed significantly earlier compared with the group without recurrent diastasis (Mann-Whitney U-test $p=0.011$ ) and the group with screw breakage had their screws significantly longer in place compared with the group without breakage $(p=0.038)$.

Conclusion: A total of 22.4 percent complications occurred upon routine removal of the syndesmotic screw. Removal might therefore be considered only in selected cases with complaints, after a minimum of eight to twelve weeks and using antibiotic prophylaxis during removal.

Keywords: Ankle fracture, Syndesmosis, Screw removal, Complications 


\section{Introduction}

In approximately one in five operatively treated ankle fractures a syndesmotic disruption necessitates surgical repair. ${ }^{6,26}$ Currently, the metallic syndesmotic positioning screw is the gold standard. Whether or not this screw needs to be removed, and after what period of time this should be done, remains debatable.

Those in favor of routine removal suggest improved outcome and range of motion upon removal and fear physical complaints of broken screws when left in place. ${ }^{14,16}$ However, current literature does not support this assumption. The improved range of motion after removal appears to be a short-term beneficial effect, as retained screw will eventually loosen. ${ }^{9,12}$ In addition, broken screws show similar outcome compared with removed screws. $^{8,12}$

In a recent review no favorable outcome was shown when syndesmotic screws were removed on a routine basis compared with screws in place, leaving the issue regarding removal of the syndesmotic screw controversial. ${ }^{23}$

In general, implant removal in adults is mainly reserved for failure or complications of the implant or in case of complaints. ${ }^{3}$ However, implant removal after ankle fractures has been associated with high wound complication rates of up to 15 percent $^{22}$ and secondly resolution of complaints can be expected in only half of the patients. ${ }^{2}$ To date, considering complications after syndesmotic screw removal, there is only information on recurrent diastasis. ${ }^{5,10,21}$

Routine removal of syndesmotic screws following operative treatment of unstable ankle fractures is currently standard practice in the Netherlands. The aim of the current study was to determine complications which accompany routine screw removal. 


\section{Patients and methods}

All consecutive patients treated for an unstable ankle fracture, between January 1, 2004 and November 30, 2010, who had a syndesmotic screw placed were included. Patients in which a bioresorbable screw was used $(n=4)$, follow-up was less than 12 weeks $(n=7)$, or in which the screw was left in place $(n=6)$ were excluded from further analysis. According to hospital protocol patients received a post-operative non-weight bearing plaster backslap for two weeks, followed by a wound check and a weight-bearing lower leg cast for four weeks. After screw removal patients were allowed full weight-bearing. Removal of syndesmotic screws is standard practice in our hospital, and protocol dictates removal between six to eight weeks as propagated by the Arbeitgemeinschaft für Osteosynthesefragen (AO) ${ }^{25}$.

Patient characteristics (i.e., age and gender), fracture characteristics (i.e., fracture side, Weber fracture type, and number of fractured malleoli), and surgical characteristics (i.e., type of anaesthesia, number of syndesmotic screws used, tri- or quadricortical placement, and delay time to screw removal) were recorded from the patient files (i.e., clinical charts and operation reports), and the picture archiving and communication system (PACS Kodak Carestream, Rochester, NY). Complications were collected from the outpatient department charts. Complications recorded were: 1) minor or major wound infection, 2) recurrent syndesmotic diastasis secondary to removal of the syndesmotic screw, and 3) removal of a broken screw. Concerning the latter, a broken screw was not considered a complication in itself, however detection of a broken screw at surgery was considered a complication, as removal would subject patients to potential surgical and anaesthetics risk, without contributing to an additional improvement in motion or outcome ${ }^{8,23}$, making the removal procedure superfluous. Recurrent diastasis was defined as widening of the syndesmosis of more than $2 \mathrm{~mm}$ compared with radiographs taken shortly after the first operation, in which the fracture was fixed, or a widening of the medial clear space of more than $2 \mathrm{~mm}$ compared with the distance between the tibia plafond (pilon) and talus., ${ }^{40,15}$ Infectious wound 
complications were divided into superficial or minor and deep or major infectious complications by applying the criteria of the Centers for Disease Control and Prevention $(C D C)$ for defining a surgical site infection. ${ }^{11,19}$ Minor complications were defined as a superficial infection treatable with conservative management like oral antibiotics only, without the need for intervention or re-admission. Major complications were defined as a deep infection in need for intervention or re-admission, like intravenous antibiotics, removal of hardware, wound debridement with or without vacuum assisted closure. The differentiation between superficial and deep infection was made by the surgeon or attending physician. IRB approval was given prior to the study.

\section{Data analysis}

The statistical analysis was performed using the Statistical Package for the Social Sciences (SPSS) version 16.0 (SPSS, Chicago, IL). The Kolmogorov-Smirnov test was used to test for normality of the data. The Levene's test was applied to assess homogeneity of variance between data. A Mann-Whitney U-test (non-parametric, numeric data), Student's T-test (parametric, numeric data) or Chi2 analysis (categoric data) was performed in order to assess statistical significance between groups with and without complications. Numeric data are expressed as mean with standard deviation (SD); categoric data are shown as numbers with percentages. Multivariable logistic regression analysis was performed in order to model the relationship between different covariates and the occurrence of recurrent dislocation (dependent variable). A similar model was made to model the relation between covariates and screw breakage. A p-value $<0.05$ was taken as level of statistical significance in all statistical tests. All calculations were made by an independent statistician. 


\section{Results}

A total of 76 patients, treated for an unstable ankle fracture, had their syndesmotic screw removed during the seven year study period. There were 48 male $(63.2 \%)$, and the right ankle was injured in $43(56.6 \%)$ of the cases. The mean age at the time of trauma was $42.6 \pm$ 17.6 years (range 14.2 to 77.1 years). Concerning the type of fracture according to the Weber classification there were 23 Weber-B fractures and 50 Weber-C type fractures. In addition, one patient had an isolated posterior malleolar avulsion fracture and two had an isolated syndesmotic ruptures. Forty-three fracture-patterns were unimalleolar, 18 were bimalleolar, 13 trimalleolar, and in two cases with isolated syndesmotic ruptures no malleolli were fractured. Seventeen patients had two screws placed (almost all in Maisonneuve type fracture) and 59 had a single screw placed. The syndesmotic screw was placed tricortically in 60 cases and quadricortically in sixteen cases. In the overall population the average time the syndesmotic screw was in place for $11.8 \pm 20.9$ weeks (range 5.0 to 181.9 weeks).

\section{Complications}

A wound infection developed in seven patients, was not found in 61 patients, and for the remaining eight patients no conclusive information could be found in the patient records. This gave a wound infection rate of 9.2 percent for the entire group and 10.3 percent when the missing cases were left out. Of the seven cases with infections, five were superficial and were adequately treated with oral antibiotics only. Two other patients developed deep infections which needed early remaining implant removal and surgical debridement. In five patients recurrent syndesmotic diastasis was visible on a control radiograph, however, in twenty cases no control radiograph following syndesmotic screw removal was available, leading to a recurrent syndesmotic diastasis rate of $6.6 \%$ for the entire group and 8.9 percent if the cases with missing radiographs were excluded.

During the surgical procedure to remove the syndesmotic screw five $(6.6 \%)$ screws appeared to be broken (Figure 1). 


\section{Statistical analysis}

Univariate analysis showed a significant association between the time of removal and recurrent syndesmotic diastasis. In patients with recurrent syndesmotic diastasis the syndesmotic screw was removed after a mean time of $6.7 \pm 7.3$ weeks (range 6.1 to 7.0 ), which was statistically significantly earlier than in patients that did not develop recurrent syndesmotic diastasis (9.7 \pm 7.3 weeks (range 5.0 to 49.9; Mann-Whitney $U$, MWU-test $p=$ 0.0011) (Figure 2).

Concomitantly, there was a significant association with the time the syndesmotic screw was in place and screw breakage. In cases without screw breakage the screws were in place for an average of $10.8 \pm 2.6$ (range 8.0 to 14.1 ) weeks compared to 11.9 (range 5.0 to 181.9) weeks in cases with screw breakage (MWU test $p=0.038)$ (Figure 2).

The multivariate analysis showed that none of the covariates (i.e., patient age and gender, Weber fracture type, number of fractured malleoli, number of syndesmotic screws used, and tri- or quadricortical placement) significantly affected the results of the univariate analyses shown above. 


\section{Discussion}

This is the first study investigating complications accompanying the routine removal of the syndesmotic screw after treatment of unstable ankle fractures. Between 6.6 and 10.3 percent wound complications occurred, between 6.6 to 10.9 percent recurrent diastasis, and in 6.6 percent broken screws were encountered at the time of removal.

Several studies caution against the routine removal of implants after fracture repair., ${ }^{3,18}$ Syndesmotic screw removal is associated with potentially high complication rates ${ }^{22}$; moreover, in approximately half of the patients it cannot be predicted if removal will result in improved functional outcome. ${ }^{2,17} \mathrm{~A}$ third argument against routine screw removal is the large amount of resources needed (operation room time) and economic costs (e.g., secondary surgery, time from work, treatment of complications). ${ }^{1}$

In the literature, dealing with implant removal following ankle fractures, infectious complications range from zero to approximately 15 percent. ${ }^{20,22}$ However, these studies do not specify the nature of the implant which was removed. One study dealing only with removal of fibular plates did not report on wound complications. ${ }^{2}$

Only one, recent, study on the occurrence of recurrent diastasis following syndesmotic screw removal is available. ${ }^{10}$ Herein, three groups who had their syndesmotic screw removed at different time intervals (6 weeks, 3 months and 9 months) were compared. In the first two groups recurrent diastasis was seen in approximately 15 percent, compared with zero percent in the third group. ${ }^{10}$ The groups were too small to detect a significant difference.

The current study shows that early removal results in an increased chance of recurrent diastasis. The percentage of recurrent diastasis between 6.6 and 8.9 in this study compares favorably to the 11.5 percent found for the entire population of 52 patients investigated by 
Hsu et al. ${ }^{10}$ Screw breakage was seen only in groups two and three in the study by Hsu, which corresponds to our findings that the longer the screws stay in place the higher the risk of breakage. $^{10}$

Serious adverse events not included in the current analysis were one superficial peroneal nerve injury detected after removal of the syndesmotic screw and one cardiac arrest successfully reanimated during surgery in a 19-year old healthy male patient under regional anesthesia.

The main limitation of this study lies in its retrospective nature, and as syndesmotic screw removal is currently standard practice in most hospitals in the Netherlands no control group was available. The number of missing values was almost 17 percent for the outpatient department charts and slightly over 26 percent for radiographs post removal of the syndesmotic screw. When patients with missing value were excluded from analysis the percentage of wound infection would rise to $10.3 \%$ and the rate of recurrent diastasis would increase to $8.9 \%$. The post-operative reduction of the syndesmosis during the initial operation was not assessed in the current study, however, it was judged on conventional radiographs (lateral and mortise views) at the outpatient department during follow-up. It is known from the literature that syndesmotic reduction might not be adequately estimated from these conventional images ${ }^{7,24,27}$. Whether or not assessing reduction with follow-up CT-scan would have influenced the number of recurrent diastasis is unknown. In any future prospective study investigating complications following the removal of the syndesmotic screw data on pre-operative use of antibiotics should be included. It is currently not routine practice in the Netherlands to administer antibiotics in implant removal surgery. In the current study it was mostly inconclusive whether or not pre-operative antibiotics were administered, especially when additional implants (i.e., plate and screws) remained in place after syndesmotic screw removal. Until now, the local hospital protocol did not include routine radiographs following syndesmotic screw removal; this has been adapted in light of the findings of this study. 


\section{Conclusions}

A total of 22.4 percent complications occurred upon routine removal of the syndesmotic screw performed as elective surgery. Literature data imply that syndesmotic screw removal will most likely not lead to improved outcome. Removal should therefore be performed only in case of complaints after a minimum of eight to twelve weeks using antibiotic prophylaxis.

The following recommendations can be made based upon the current study and related literature:

1. Removal of the syndesmotic screw might be advisable only in case of complaints related to the implant ${ }^{23}$

2. In case removal is considered, it should be performed after a minimum of eight weeks ${ }^{10,13}$

3. Antibiotic prophylaxis should be considered upon removal ${ }^{22}$

4. Radiographs should be taken shortly prior to removal in order to identify broken screws and to prevent unnecessary surgery

5. Control radiographs following removal should be routinely made for the identification of recurrent diastasis 


\section{References}

1. Bostman, O., and Pihlajamaki, H.: Routine implant removal after fracture surgery: a potentially reducible consumer of hospital resources in trauma units. J Trauma, 41(5): 846-9, 1996.

2. Brown, O. L.; Dirschl, D. R.; and Obremskey, W. T.: Incidence of hardwarerelated pain and its effect on functional outcomes after open reduction and internal fixation of ankle fractures. J Orthop Trauma, 15(4): 271-4, 2001.

3. Busam, M. L.; Esther, R. J.; and Obremskey, W. T.: Hardware removal: indications and expectations. J Am Acad Orthop Surg, 14(2): 113-20, 2006.

4. Chissell, H. R., and Jones, J.: The influence of a diastasis screw on the outcome of Weber type-C ankle fractures. J Bone Joint Surg Br, 77(3): 435-8, 1995.

5. Donatto, K. C.: Ankle fractures and syndesmosis injuries. Orthop Clin North Am, 32(1): 79-90, 2001.

6. Egol, K. A.; Pahk, B.; Walsh, M.; Tejwani, N. C.; Davidovitch, R. I.; and Koval, K. J.: Outcome after unstable ankle fracture: effect of syndesmotic stabilization. $J$ Orthop Trauma, 24(1): 7-11, 2010.

7. Gardner, M. J.; Demetrakopoulos, D.; Briggs, S. M.; Helfet, D. L.; and Lorich, D. G.: Malreduction of the tibiofibular syndesmosis in ankle fractures. Foot Ankle Int, 27(10): 788-92, 2006.

8. Hamid, N.; Loeffler, B. J.; Braddy, W.; Kellam, J. F.; Cohen, B. E.; and Bosse, M. J.: Outcome after fixation of ankle fractures with an injury to the syndesmosis: the effect of the syndesmosis screw. J Bone Joint Surg Br, 91(8): 1069-73, 2009.

9. Heim, D.; Schmidlin, V.; and Ziviello, O.: Do type B malleolar fractures need a positioning screw? Injury, 33(8): 729-34, 2002.

10. Hsu, Y. T.; Wu, C. C.; Lee, W. C.; Fan, K. F.; Tseng, I. C.; and Lee, P. C.: Surgical treatment of syndesmotic diastasis: emphasis on effect of syndesmotic screw on ankle function. Int Orthop, 35(3): 359-64, 2011.

11. Mangram, A. J.; Horan, T. C.; Pearson, M. L.; Silver, L. C.; and Jarvis, W. R.: Guideline for Prevention of Surgical Site Infection, 1999. Centers for Disease Control and Prevention (CDC) Hospital Infection Control Practices Advisory Committee. Am J Infect Control, 27(2): 97-132; quiz 133-4; discussion 96, 1999.

12. Manjoo, A.; Sanders, D. W.; Tieszer, C.; and MacLeod, M. D.: Functional and radiographic results of patients with syndesmotic screw fixation: implications for screw removal. J Orthop Trauma, 24(1): 2-6, 2010.

13. Manjoo, A.; Sanders, D. W.; Tieszer, C.; and MacLeod, M. D.: Functional and radiographic results of patients with syndesmotic screw fixation: implications for screw removal. J Orthop Trauma, 24(1): 2-6.

14. Melvin, J. S.; Downing, K. L.; and Ogilvie, C. M.: A technique for removal of broken cannulated tricortical syndesmotic screws. J Orthop Trauma, 22(9): 648-51, 2008.

15. Michelson, J. D.: Fractures about the ankle. J Bone Joint Surg Am, 77(1): 142-52, 1995.

16. Miller, A. N.; Paul, O.; Boraiah, S.; Parker, R. J.; Helfet, D. L.; and Lorich, D. G.: Functional outcomes after syndesmotic screw fixation and removal. J Orthop Trauma, 24(1): 12-6, 2010.

17. Minkowitz, R. B.; Bhadsavle, S.; Walsh, M.; and Egol, K. A.: Removal of painful orthopaedic implants after fracture union. J Bone Joint Surg Am, 89(9): 1906-12, 2007. 
18. Muller-Farber, J.: [Removal of metal in traumatology]. Unfallchirurg, 106(8): 65368, 2003.

19. Petherick, E. S.; Dalton, J. E.; Moore, P. J.; and Cullum, N.: Methods for identifying surgical wound infection after discharge from hospital: a systematic review. BMC Infect Dis, 6: 170, 2006.

20. Richards, R. H.; Palmer, J. D.; and Clarke, N. M.: Observations on removal of metal implants. Injury, 23(1): 25-8, 1992.

21. Roberts, R. S.: Surgical treatment of displaced ankle fractures. Clin Orthop Relat Res, (172): 164-70, 1983.

22. Sanderson, P. L.; Ryan, W.; and Turner, P. G.: Complications of metalwork removal. Injury, 23(1): 29-30, 1992.

23. Schepers, T.: To retain or remove the syndesmotic screw: a review of literature. Arch Orthop Trauma Surg, 2010.

24. Schwarz, N., and Köfer, E.: Postoperative Computed Tomography-Based Control of Syndesmotic Screws. Eur J Trauma, 31(3): 266-270, 2005.

25. Tile, M.: Fractures of the ankle. In The Rationale of Operative Fracture Care, pp. 580-581. Edited by Schatzker, J., and Tile, M., 580-581, New York, Springer-Verlag, 2005.

26. Van den Bekerom, M. P.; Lamme, B.; Hogervorst, M.; and Bolhuis, H. W.: Which ankle fractures require syndesmotic stabilization? J Foot Ankle Surg, 46(6): 456-63, 2007.

27. Wanders, L., and Oliver, C. W.: Fibular malreduction in AO/Weber type C ankle fractures. Injury, 29(2): 144-6, 1998. 


\section{Figures}

Figure 1. Examples of removed and retained syndesmotic screws
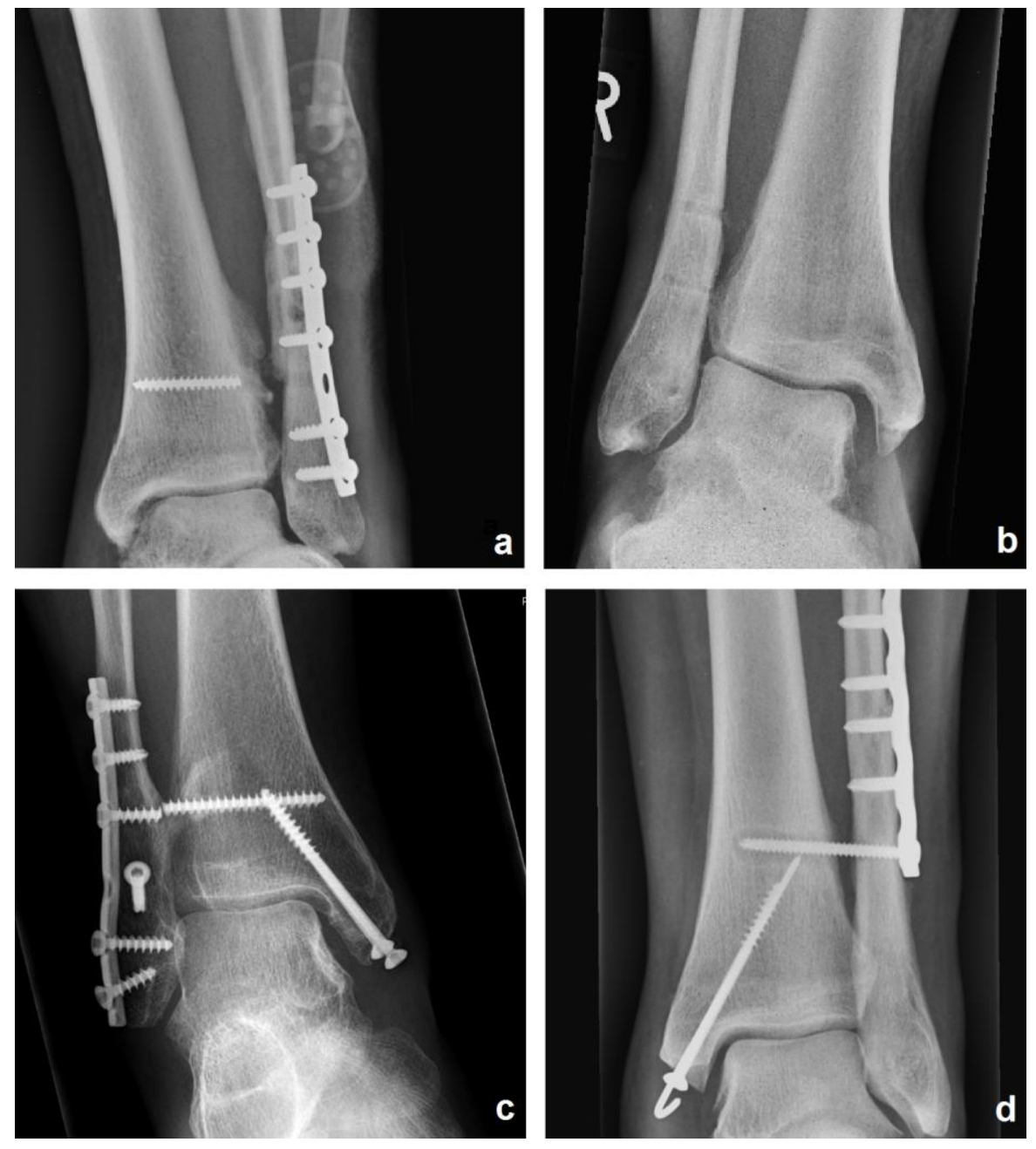

a. Deep infection following removal of a broken syndesmotic screw

b. Recurrent diastasis after two syndesmotic screws were removed

c. Retained broken syndesmotic screw without complaints

d. Retained tricortically placed syndesmotic screw showing loosening 
Figure 2. Kaplan-Meier plot showing complications following routine removal of the syndesmotic screw

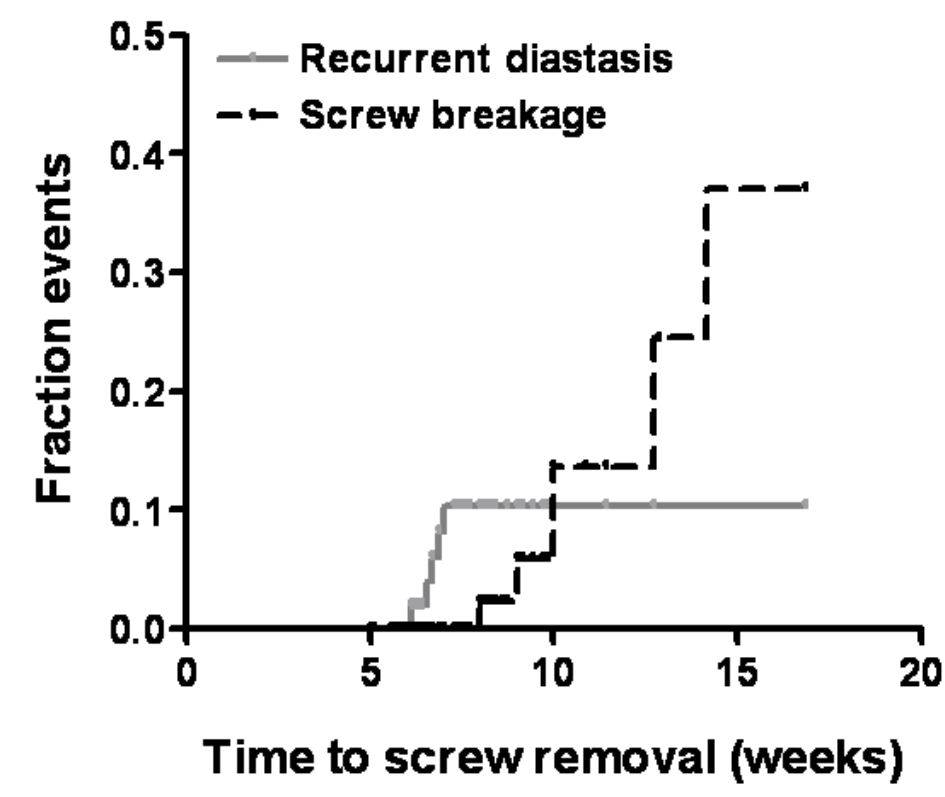

Kaplan-Meier plot showing the correlation between the time the syndesmotic screw is in place and the number of adverse events: recurrent diastasis (gray line) and screw breakage (black line) 